Elsevier

\title{
Spatial distribution of neural activity evoked by electrical stimulation of the cochlea
}

\author{
Allen F. Ryan ${ }^{1,2}$, Josef M. Miller ${ }^{3}$, Zhi-Xian Wang ${ }^{4}$ and Nigel K. Woolf ${ }^{1}$ \\ Departments of ${ }^{\prime}$ Surgery/Otolaryngology and ${ }^{2}$ Neurosciences, UCSD School of Medicine and VA Medical Center, La Jolla, California, \\ ${ }^{3}$ Kresge Hearing Research Institute, University of Michigan, Ann Arbor, Michigan, U.S.A. and ${ }^{4}$ Department of Experimental Pathologv, \\ Institute of Hygiene, Tianjin, People's Republic of China
}

(Received 11 December 1989; accepted 18 May 1990)

\begin{abstract}
Activity in the central auditory system was mapped with 2-deoxyglucose (2-DG) autoradiography, using either pure tones or electrical stimulation of the normal cochlea. Electrical stimulation with both monopolar (distant reference electrode) and bipolar prostheses near threshold increased 2-DG uptake in auditory nuclei in a manner similar to that seen with a pure tone: increased 2-DG uptake was restricted to a small frequency region of brainstem and mid-brain auditory nuclei. The position of this area was related to the cochlear location of the prosthesis. At higher current amplitudes only the bipolar prosthesis retained spatial restriction of evoked neural activity, while stimulation through a monopolar prosthesis produced evoked activity in all frequency regions of auditory nuclei, and in non-auditory nuclei. Activation of non-auditory structures was consistent with spread of current through the brainstem, rather than activation of peripheral nerves. At all current amplitudes, a monopolar prosthesis evoked higher levels of 2-DG uptake than a bipolar prosthesis.

The results suggest that while a bipolar prosthesis provides greater spatial restriction of evoked neural activity and a greater dynamic range, a monopolar prosthesis produces higher levels of evoked activity.
\end{abstract}

Cochlear prosthesis; Central auditory system; 2-deoxyglucose; Gerbil; Ear, inner

\section{Introduction}

Direct electrical stimulation of the inner ear with a cochlear prosthesis is increasingly used as a rehahilitative procedure for the profoundly deaf. The devices being used include both single electrode prostheses, referenced to a distant electrode outside of the cochlea, and multi-electrode prostheses in which current may be delivered between two closely-spaced electrodes. The latter are designed to provide multiple channels of input to the nervous system, originating at various locations along the cochlea. The response of auditory neurons to electrical stimulation is quite different than that evoked by sound. For example, the dynamic range of activation of auditory neurons

Correspondence to: Allen F. Ryan, ENT, V-112C, UCSD, La Jolla, CA 92093, U.S.A. by electrical stimulation is small when compared to that achieved with acoustic stimuli (Glass, 1983; Van den Honert and Stypulkowski, 1984; Javel et al., 1987). Moreover, with single channel prostheses, the thresholds of all neurons are similar, unlike the diversity of thresholds seen with acoustic stimuli. These response characteristics presumably limit the information-carrying capacity of a single channel, and increase the importance of providing multiple information-carrying channels for signal input into the cochlea.

At the level of the inferior colliculus (IC), single unit recordings suggest that activation of only a portion of the nucleus is possible, depending upon the arrangement of an electrode pair in the cochlea (Merzenich and White, 1977). More recent and detailed observations by Van den Honert and Stypulkowski $(1984 ; 1987)$ have corroborated these findings at the level of the VIIIth nerve. They found that a bipolar prosthesis provides a more 
discrete activation of auditory nerve fibers than a monopolar prosthesis.

These single unit studies have provided detailed information regarding the coding of stimulus information during electrical stimulation of the cochlea. However, assessment of the spatial distribution of activity in neural structures using single unit techniques is time-consuming, and usually restricted to a single structure, due to the number of observations required for a spatial map of high resolution. The 2-deoxyglucose (2-DG) autoradiographic technique (Sokoloff et al., 1977) has provided an alternative method for spatial mapping of neural activity evoked in the auditory system by acoustic stimuli (e.g. Huang and Fex, 1986; Hungerbuhler et al., 1981; Ryan and Woolf, 1988; Ryan et al., 1988; Scheich et al., 1986; Serviere et al., 1984; Sharp et al., 1981; Webster et al., 1978). Although the technique does not reveal the temporal characteristics of neural discharge, it simultaneously provides spatial maps of neural activity in all auditory nuclei in a single preparation.

The purpose of this investigation was to examine the patterns of central nervous system activation produced by two different types of cochlear prostheses, under varying conditions of stimulus current and electrode position, and to compare the results to activity evoked by acoustic stimulation.

\section{Methods}

Forty-seven adult mongolian gerbils (Meriones unguiculatus) were used as subjects. Only young adult (45-90 days old) animals were used since the gerbil develops a vesicular neuropathology in the cochlear nucleus (CN) soon after birth (Ostapoff and Morest, 1989). While this pathology has no known functional correlate, young animals were employed to minimize any possible effects. 2-deoxy-D-1 $\left[{ }^{14} \mathrm{C}\right]$-glucose $(57 \mathrm{mCi} / \mathrm{mM}$, AmershamSearle) was evaporated and reconstituted in saline. Animals were injected intraperitoneally (IP) with 16.7 UCi of 2-DG/100 gm body weight, in $100 \mu \mathrm{l}$. Acoustic stimulation was provided continuously to unanesthetized animals placed in a cylindrical cage $(20 \mathrm{~cm}$ diameter) suspended centrally in a double-walled, sound attenuated chamber (IAC $1200 \mathrm{~A}$ ) with anechoic lining, for one hour in the dark. Two subjects at each frequency were exposed a pure tone stimulus, at octave intervals from 0.375 to $48 \mathrm{kHz}$, from a speaker $90 \mathrm{~cm}$ over the animal's head. Three additional subjects were anesthetized with pentobarbital $(32 \mathrm{mg} / \mathrm{kg}$, IP) and ketamine $(25 \mathrm{mg} / \mathrm{kg}$, intramuscular) prior to IP 2-DG injection and exposure to $3,0,16.0$ or $24.0 \mathrm{kHz}$ tones. The intensity of each tone was set at $40-45 \mathrm{~dB}$ above behavioral threshold (Ryan, 1976). Frequencies were chosen to span the range of characteristic frequencies which have been reported for auditory neurons in the gerbil (Schmeidt, 1977). Stimulus generation and calibration procedures are described elsewhere (Ryan and Woolf, 1988).

For electrical stimulation, animals were anesthetized. Their bullae were exposed and opened using a lateral approach, which provided wide access to the cochlea. Cochlear prostheses consisted of either single or bipolar platinumiridium ball electrodes (200 um diameter) in a silastic carrier (Spelman et al., 1982). Electrode separation of bipolar pairs was $0.5 \mathrm{~mm}$ center-to-

TABLE I

PERCENT OF DCN AND IC AREA ACTIVATED BY ELECTRICAL STIMULATION WITH BIPOLAR VERSUS MONOPOLAR PROSTHESES (MEAN $\pm S D)$

\begin{tabular}{|c|c|c|c|c|c|c|}
\hline \multirow[b]{2}{*}{$\mu \mathrm{A}$} & \multicolumn{3}{|c|}{ Monopolar prosthesis } & \multicolumn{3}{|c|}{ Bipolar prosthesis } \\
\hline & DCN & IC & $(N)$ & DCN & IC & $(N)$ \\
\hline 25 & $0.0 \pm 0.0$ & $0.0 \pm 0.0$ & (2) & $0.0 \pm 0.0$ & $0.0 \pm 0.0$ & (2) \\
\hline 50 & $25.2 \pm 12.8$ & $6.2 \pm 1.8$ & (2) & $11.1 \pm 1.6$ & $5.2 \pm 2.3$ & (2) \\
\hline 100 & $67.0 \pm 5.7$ & $31.8 \pm 0.9$ & (2) & $16.3 \pm 7.5$ & $7.1 \pm 4.9$ & (5) \\
\hline 200 & $63.3 \pm 5.0$ & $32.4 \pm 4.7$ & (2) & $16.5 \pm 4.8$ & $5.9 \pm 3.1$ & (4) \\
\hline 400 & $73.5 \pm 0.9$ & $39.1 \pm 5.0$ & (2) & $22.5 \pm 2.1$ & $11.6 \pm 1.0$ & (2) \\
\hline
\end{tabular}


center. Prostheses were inserted into the opened round window, or into holes which were handdrilled through the lateral wall in the upper cochlear turns. Prostheses were activated with a 1.0 $\mathrm{kHz}$, charge-balanced, constant-current, sinusoidal stimulus. Current levels were varied from 25 to $400 \mu \mathrm{A}$. For the monopolar prosthesis, a low-impedance indifferent electrode was placed on the neck musculature. The numbers of animals employed for each current with each prosthesis type are given in Table $\mathrm{I}$.

Immediately following the one hour exposure to tone or electrical stimulation, subjects were instantaneously decapitated and their brains rapidly dissected and frozen in isopentane cooled to $-30^{\circ} \mathrm{C}$. After cooling to $-70^{\circ} \mathrm{C}$ on dry ice, the brains were coated with thin layers of Lipshaw embedding matrix and serially sectioned in the transverse plane on an American Optical cryostat at a section thickness of $20 \mu \mathrm{m}$. All sections were mounted on coverslips and rapidly dried on a hotplate at $60^{\circ} \mathrm{C}$. Sections were exposed on Kodak SB-5 X-ray film for 7 days, developed in Kodak $X$-ray film developer for $5 \mathrm{~min}$, washed $2 \mathrm{~min}$, fixed in Kodak rapid-fix for $5 \mathrm{~min}$, washed $20 \mathrm{~min}$ and air-dried.

Restricted regions of very high radioactivity (compared to that of the adjacent tissue within the nucleus) were readily apparent in the autoradiographs of most subjects. This was identified as evoked 2-DG uptake.

A pure tone of low-to-moderate intensity activates a restricted volume of an auditory nucleus. Given a series of pure tones spanning the audible range, a tonotopic axis can be defined in an auditory structure as a line running through the centers of such nuclear volumes. This axis approximately parallels the transverse plane of the dorsal cochlear nucleus (DCN) and inferior colliculus (IC). We therefore restricted quantitative analysis to these nuclei.

Autoradiographic images were digitized on a Drexel University image analysis system. This permitted the measurement of optical densities, generation of optical density profiles, and objective identification of tissue areas which exceeded a criterion optical density. A criterion of $50 \%$ of the difference between the background of a nucleus and the peak optical density evoked by $k+$ stimu- lation was employed to define the boundaries of regions of evoked 2-DG uptake. This criterion closely matches the visually identified boundary of evoked 2-DG uptake. The extent of spatial activation of the DCN and IC was analyzed from sections at the center of the anterior-to-posterior extent of each nucleus, by measuring the percentage of the area of each nucleus which exceeded the $50 \%$ criterion optical density.

For optical density measurements, the image analyzer was calibrated using optical density standards (Kodak) and the densities of images from the ${ }^{14} \mathrm{C}$ standards (New England Nuclear) were read to assure that the images of brain structures were not saturated. The optical density for each structure was read on six adjacent sections. The following structures had optical densities determined on the left and right sides: dorsal cochlear nucleus ( $\mathrm{DCN}$ ), posterior ventral cochlear nucleus (PVCN), inferior colliculus (IC), vestibular nuclei (VN), trigeminal nuclei (TN) and facial motor nucleus (FN). For each nucleus on the side innervated by the stimulated cochlea, that portion of the image which exhibited evoked metabolic activity was measured. If no activity was present, the entire structure was measured. The equivalent area of structure on the opposite, unstimulated side was also measured. The optical density of the anterior inferior cerebellar peduncle (ICP) at the level of the DCN was also measured bilaterally.

Optical density measurements were analyzed as follows. For each section, the background optical density of the film was subtracted from all structure densities. The optical density of each gray matter structure was then divided by the averaged optical density of the ICP, to obtain an optical density ratio. Such ratios of gray matter to white matter were used to help control for small variations in section thickness, exposure and development times, and tracer dose (Sharp et al., 1983). They provide a measure of relative 2-DG uptake which we have used previously to demonstrate stimulus-related auditory neural activity (Sharp, et. al., 1981; Ryan and Woolf, 1988; Ryan, et al., 1982,1988 ). The optical density ratio from the unstimulated side was then subtracted from that of the stimulated side for each section. This difference provided a normalized measure of stimulation effects for each structure. 
Raw optical densities were analyzed by a paired $t$-test. Normalized optical densities were analyzed by the Mann-Whitney U-test. All subjects in each stimulus condition were analyzed.

\section{Results}

\section{Acoustic stimulation}

In the $\mathrm{CN}$, superior olive, nuclei of the lateral lemniscus and IC, pure tone stimulation produced 2-DG uptake which was markedly higher than in adjacent tissue. Pure tones produced less dramatic but recognizable, responses in the medial geniculate nucleus and auditory cortex. This evoked 2-DG uptake occurred in a restricted region of each nucleus, and the location of this region varied systematically with frequency. In the $D C N$, for example, $375 \mathrm{~Hz}$ stimulation produced a restricted region of increased 2-DG uptake at the ventrolateral edge of the nucleus. Increasing frequencies of stimulation produced increased metabolic activity at progressively more dorsomedial locations, until a $48 \mathrm{kHz}$ stimulus produced a small area of evoked 2-DG uptake at the dorsomedial edge of the DCN. For all frequencies, 2-DG uptake was seen in the deep DCN and fusiform cell layers, but not in the molecular cell layer. Sound-evoked 2-DG uptake in the IC is illustrated in Fig. 1. As can be seen, low frequency stimulation activated a band of tissue in the dorsolateral pole of the central nucleus and the medial half of the external cortex. With increasing frequency of stimulation the lamina of activated tissue was located in progressively more ventromedial locations, until the $48 \mathrm{kHz}$ tone produced a band of high activity at the ventromedial margin. No activity was evoked in the brachium of the IC, or in the dorsal cortex. At the stimulation level used, constant intensity re behavioral threshold, the area of activated tissue tended to decrease with increasing stimulus frequency. At $48 \mathrm{kHz}$, a secondary band of activity was also present. The spatial response to pure tones in anesthetized animals was identical to that seen in unanesthetized subjects, although the overall levels of 2-DG uptake in the higher auditory nuclei were depressed as we have previously reported with broad band signals (Wang et al., 1987).

\section{Monopolar cochlear prosthesis}

The upper panel of Fig. 2 illustrates the patterns of $\mathrm{CN}$ and $\mathrm{IC}$ tissue activation observed in autoradiographs after stimulation with a monopolar prosthesis in the basal turn with increasing current amplitude. At $25 \mu \mathrm{A}$ (not illustrated), there was no observable effect of stimulation in any structure. At $50 \mu \mathrm{A}$, stimulation produced a discrete band of increased 2-DG in the dorsomedial aspects of the DCN and PVCN, and in the ventromedial aspect of the IC. This location was consistent with the position of the electrode in the cochlea. However, there also appeared to be some activation of neural tissue in the ventrolateral $\mathrm{CN}$ and dorsolateral $\mathrm{IC}$, areas which receive input from more apical cochlear locations. At $100 \mu \mathrm{A}$, increased 2-DG uptake was seen throughout the $\mathrm{CN}$ and $\mathrm{IC}$, in all tissue areas which respond to broad-band acoustic stimulation (Sharp et al., 1981). Elevated uptake was also apparent in the VN. At $200 \mu \mathrm{A}$, uptake in the $\mathrm{CN}$ and IC was comparable to that seen at $100 \mu \mathrm{A}$. Increased 2-DG uptake was also apparent in the VN, TN and FN. The response observed at $400 \mu \mathrm{A}$ (not illustrated) was similar to that observed at 200 $\mu \mathrm{A}$, except that the amplitude of response in the non-auditory structures was greater. Even at the highest stimulus amplitude, areas of auditory nuclei which do not respond to acoustic stimulation (such as the molecular layer of the $\mathrm{DCN}$ ) also did not respond to electrical stimulation of the cochlea.

The lower panel of Fig. 2 illustrates optical density profiles obtained along the tonotopic axis in the IC, at stimulus intensities from 25 to 400 $\mu \mathrm{A}$. At $50 \mu \mathrm{A}$, the region of evoked 2-DG uptake was apparent as a peak in the ventromedial IC, occupying approximately $20 \%$ of the tonotopic axis. From 100-400 $\mu \mathrm{A}$, increased 2-DG uptake occupied the entire axis.

Increased 2-DG uptake was not observed in either the medial geniculate nucleus or auditory cortex at any current level, consistent with previous observations in anesthetized animals using acoustic stimulation (Wang et al., 1987).

The upper panel of Fig. 3 illustrates the resuits of optical density measurements of 2-DG uptake in the DCN, PVCN and IC, after stimulation with a monopolar prosthesis at five current amplitudes. 


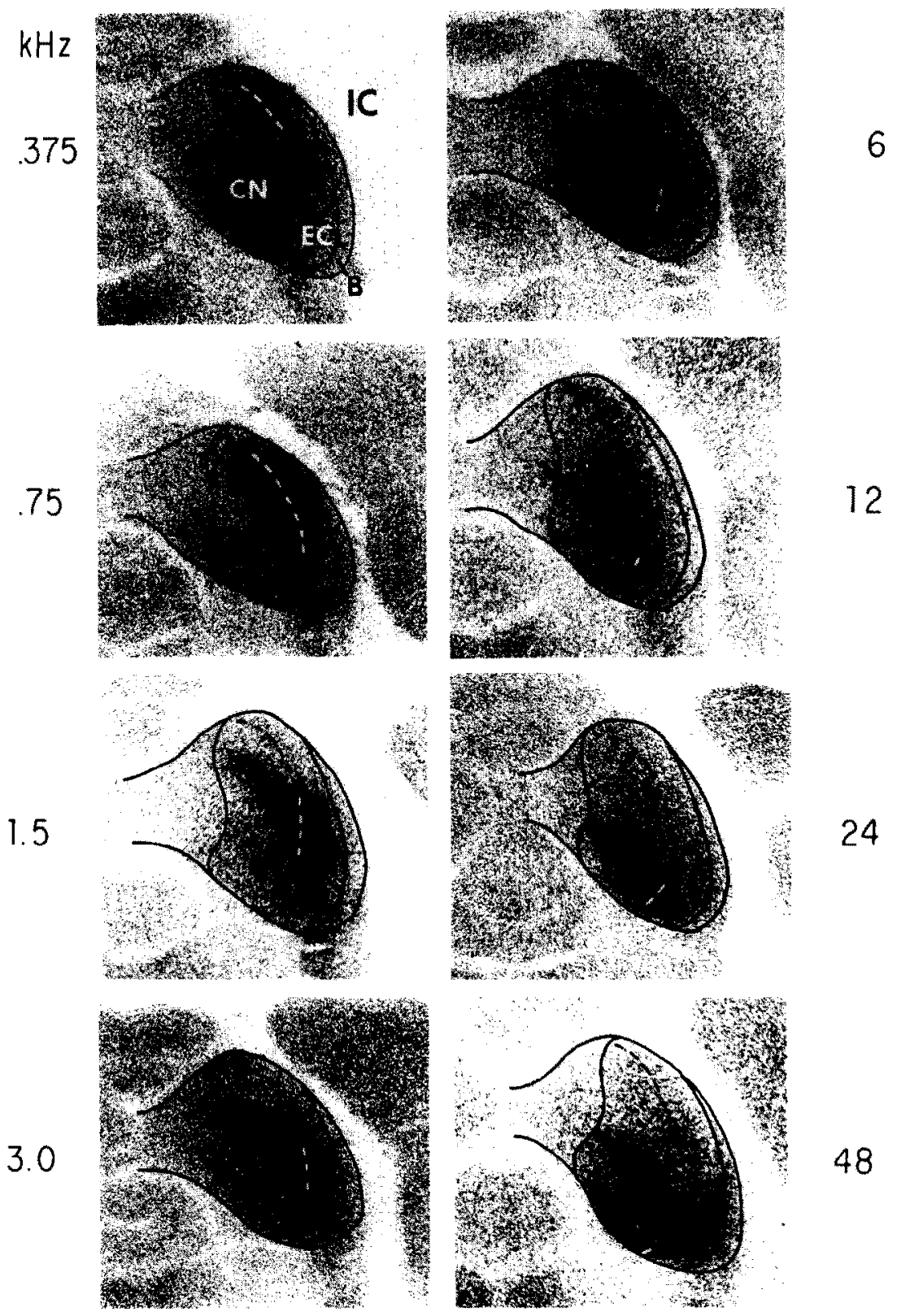

Fig. 1. Typical patterns of 2-DG uptake produced by pure tone stimulation in the gerbil IC. Each tone, presented at $40-45 \mathrm{~dB}$ above behavioral threshold (Ryan, 1976), produced a restricted band of evoked 2-DG uptake which was much higher than the background of the nucleus. Bands were oriented dorsomedial to ventrolateral across the central nucleus (CN) and part of the external cortex (EC) of the IC. The location of this region varied systematically with frequency.

There was no difference in 2-DG uptake between the stimulated and unstimulated sides of the brain at $25 \mu \mathrm{A}$. At $50 \mu \mathrm{A}$, a significant increase $(P<$ 0.01 ) in 2-DG uptake was observed on the stimulated side, in all three auditory nuclei. The highest level of evoked uptake was observed in the stimulated PVCN, the next highest in the $\mathrm{DCN}$, and the lowest in the IC. Similar increases were observed at stimulation currents from 100 to $400 \mu \mathrm{A}$.

The lower panel of Fig. 3 shows the responses 

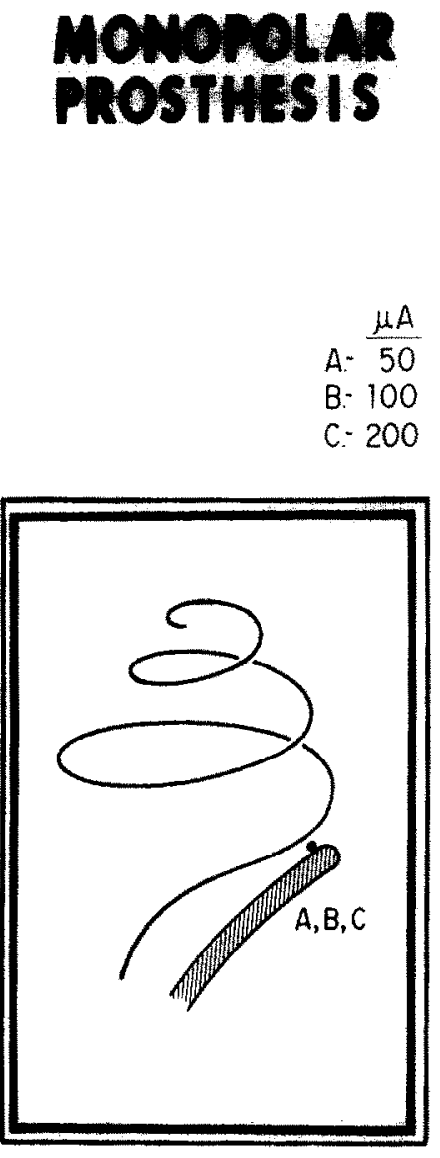

$25 \mu \mathrm{A}$

$50 \mu \mathrm{A}$

$100 \mu A$
$200 \mu A$

$400 \mu \mathrm{A}$

A.
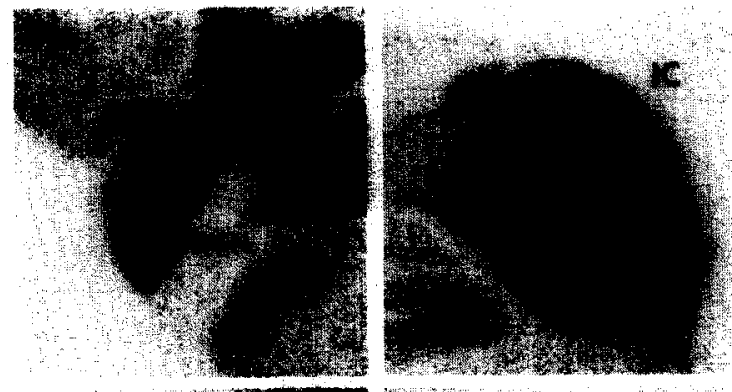

B.

C.
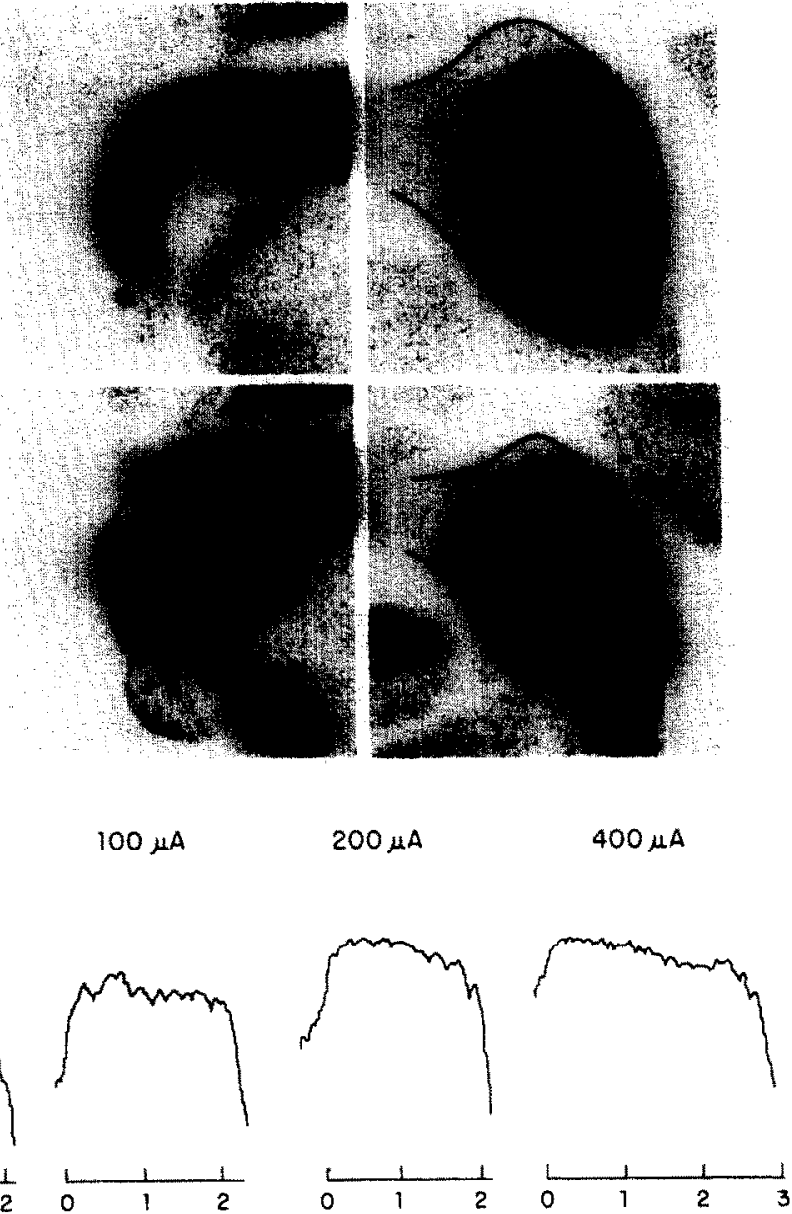

OISTANCE $(\mathrm{mm}) \mathrm{VM}-\mathrm{DL}$

Fig. 2. (Upper panel): Typical patterns of 2-DG uptake evoked with a monopolar prosthesis in the basal tum approximately $2 \mathrm{~mm}$ above the round window, in the posterior $\mathrm{CN}$ and IC. The active electrode was placed in scala tympani, just beneath the osseous spiral lamina. The indifferent electrode was placed on the neck musculature. (Lower panel): Optical density scans along the tonotopic axis of the IC (indicated by the straight line in the $50 \mu \mathrm{A}, \mathrm{IC}$ autoradiograph of the upper panel) after stimulation with a monopolar cochlear prosthesis at stimulus levels of 25 to $400 \mu \mathrm{A}$. A localized peak of activity was observed only at $50 \mu \mathrm{A}$. 


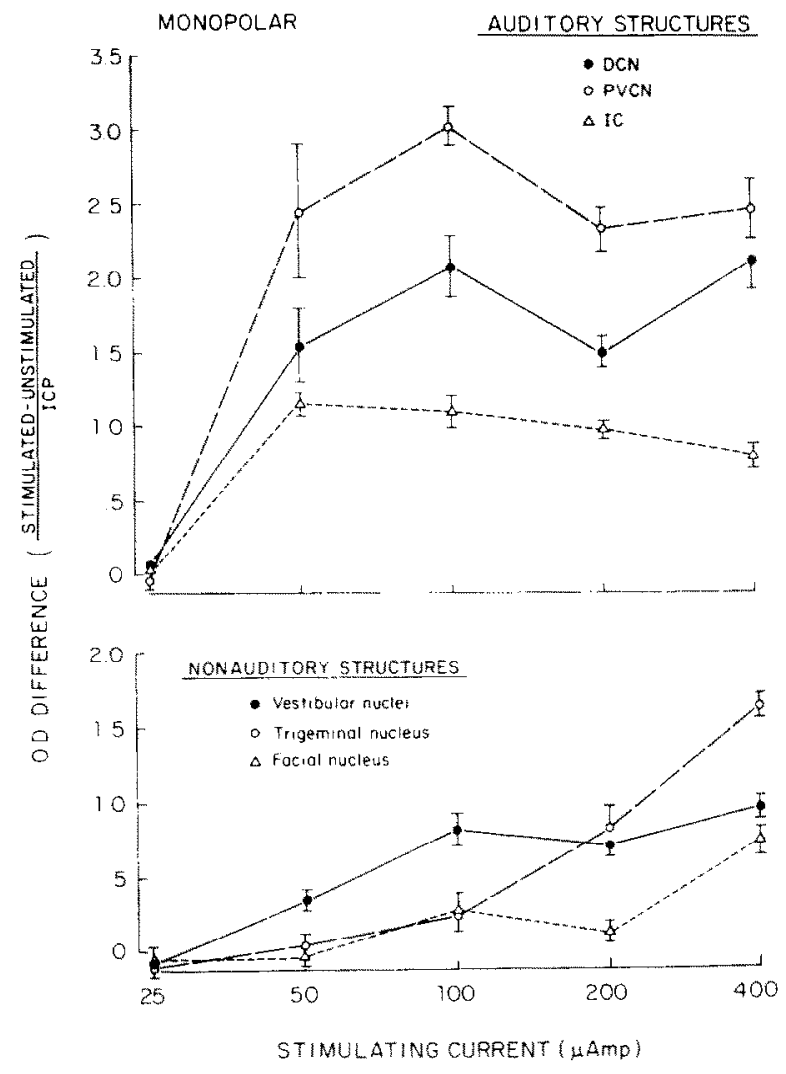

Fig. 3. Evoked 2-DG uptake produced by stimulation with a monopolar cochlear prosthesis at five current levels. The data are expressed as the optical density difference measured between each nucleus on the stimulated and unstimulated sides of the brain. (Upper panel): In three auditory nuclei, evoked uptake saturated at $50 \mu \mathrm{A}$. (Lower panel): A monopolar prosthesis produced increased levels of evoked 2-DG uptake in all three structures.

of three non-auditory nuclei: the VN, TN and FN, to stimulation delivered through a monopolar prosthesis. None of the structures showed evidence of evoked activity at $25 \mu \mathrm{A}$. Relative $2-\mathrm{DG}$ uptake was significantly increased $(P<0.05)$ in the VN on the stimulated side at $50 \mu \mathrm{A}$, and in all three nuclei at $100 \mu \mathrm{A}$. The evoked increase in 2-DG uptake saturated at $100 \mu \mathrm{A}$ for the $\mathrm{VN}$, but increased with increasing stimulus current in the TN and FN.

Table I illustrates the percentages of the area of the DCN and IC which were activated by stimulation of the basal turn through a monopolar prosthesis, measured from sections at the center of the A-P extent of each nucleus. Stimulation at 50 $\mu \mathrm{A}$ activated a small area within each nucleus. Increasing stimulus current produced rapid spread of activity, reaching an asymptote which represents all responsive tissue in each nucleus, at 100 $\mu \mathrm{A}$.

\section{Bipolar cochlear prosthesis}

The upper panel of Fig. 4 illustrates the effects of stimulation through a bipolar prosthesis in the basal turn, at five intensities from 25 to $400 \mu \mathrm{A}$. The $25 \mu \mathrm{A}$ stimulus did not produce obvious increases in 2-DG uptake. At $50 \mu \mathrm{A}$ increased 2-DG uptake was observed in discrete areas of the dorsomedial DCN and PVCN, and in the ventromedial IC. With increasing amplitude of stimulation, increasing tissue area of 2-DG uptake was noted within these auditory nuclei. However. even at $400 \mu \mathrm{A}$ the area of tissue exhibiting increased 2-DG uptake remained restricted to a relatively small proportion of each nucleus. At these higher intensities, in the IC, the area of evoked 2-DG uptake displayed two parallel bands.

The lower panel of Fig. 4 illustrates optical density profiles obtained along the tonotopic axis of the IC, for basal turn stimulation through a bipolar prosthesis at varying currents. While no evoked 2-DG uptake was observed at $25 \mu \mathrm{A}$, a restricted region of uptake was apparent as a simple peak in the profile at 50 and $100 \mu \mathrm{A}$. At both intensities, the peak occupied approximately $20 \%$ of the tonotopic axis. At $200 \mu \mathrm{A}$, a double peak was apparent, again occupying $20 \%$ of the axis. At $400 \mu \mathrm{A}$, the peak had spread to about $35 \%$ of the axis.

The upper panel of Fig. 5 illustrates 2-DG uptake in the $\mathrm{CN}$ and IC observed with stimulation through a bipolar prosthesis at each of four sites along the cochlea. The amplitudes of stimulation used at each site were near threshold for clearly observed evoked 2-DG uptake. Apical sites required a higher amplitude of stimulation. In each case, stimulation produced a discrete pattern of tissue activation in the $\mathrm{CN}$ and IC. As the stimulation site varied from the base to the apex, the location of activated neural tissue moved in a systematic fashion from dorsomedial to ventrolateral in the DCN and PVCN, and ventromedial to dorsolateral in the IC. However, in the second turn placement (Fig. $5 \mathrm{C}$ ) neural tissue was 


\section{dipolar mogtwests}

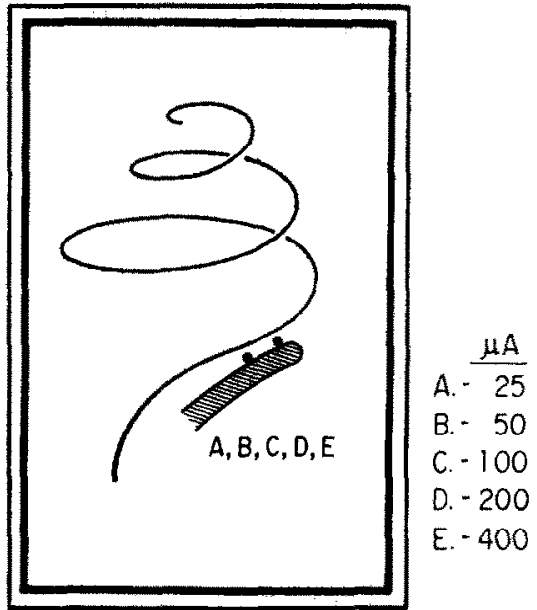

A.
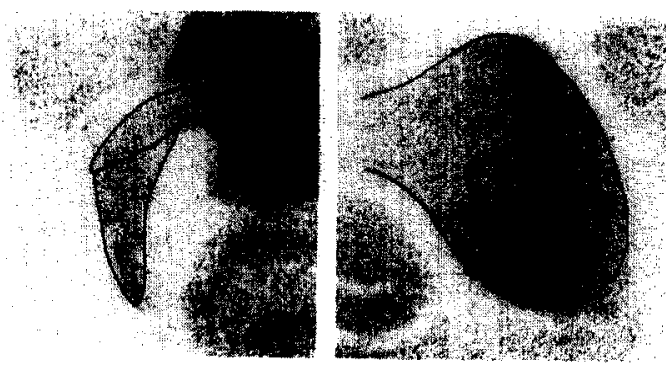

$25 \mu \mathrm{A}$

$50 \mu \mathrm{A}$

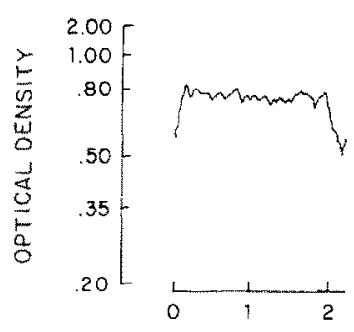

B.
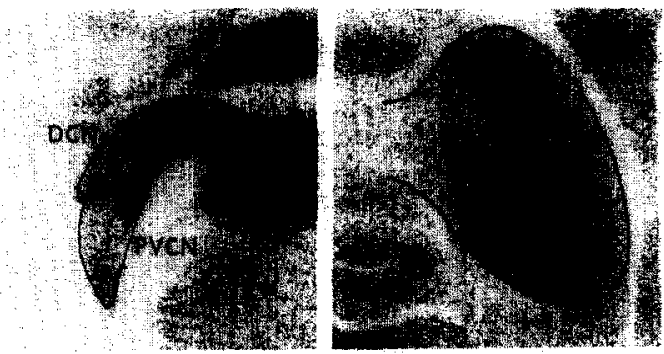

C.

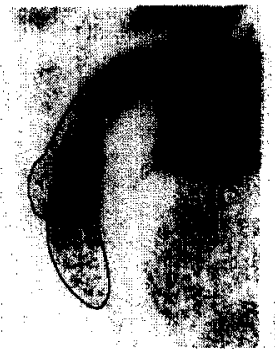

D.

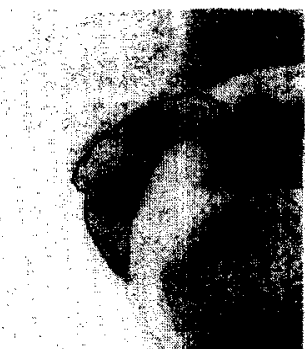

E.
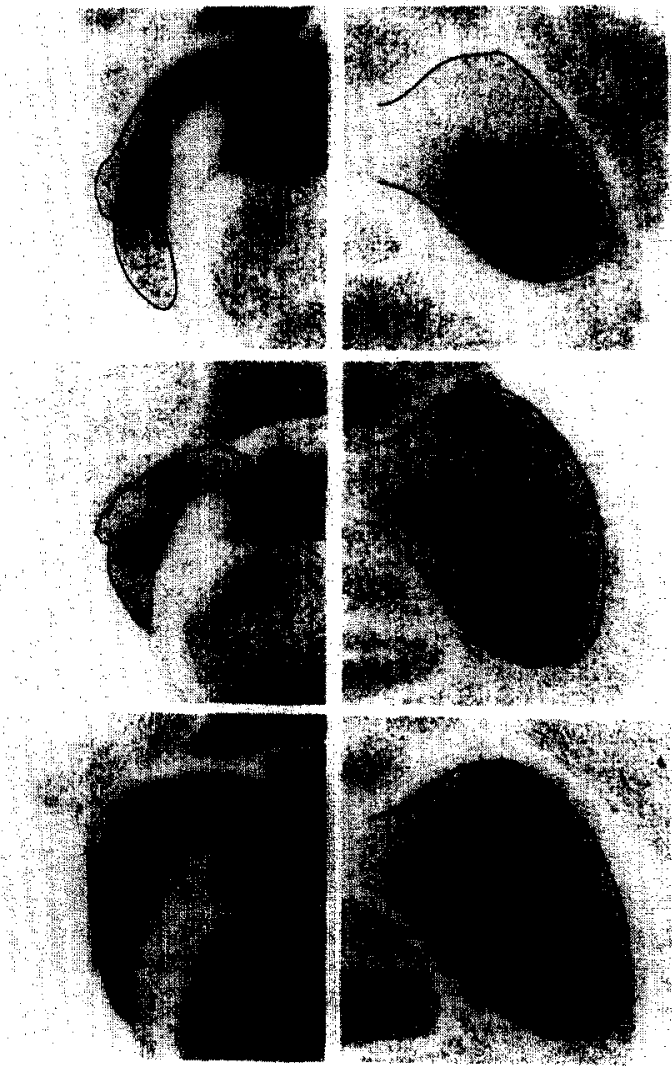

Thents
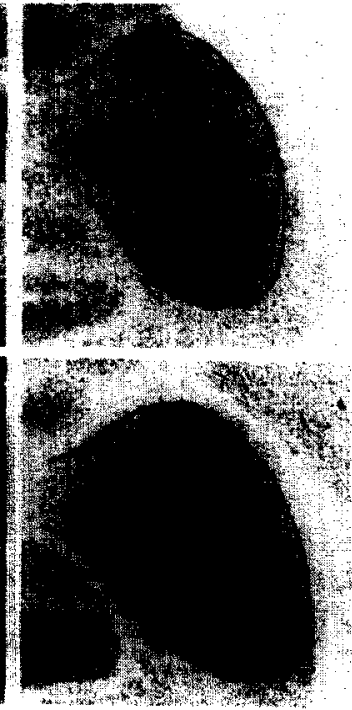

$100 \mu \mathrm{A}$

$200 \mu \mathrm{A}$

$400 \mu \mathrm{A}$
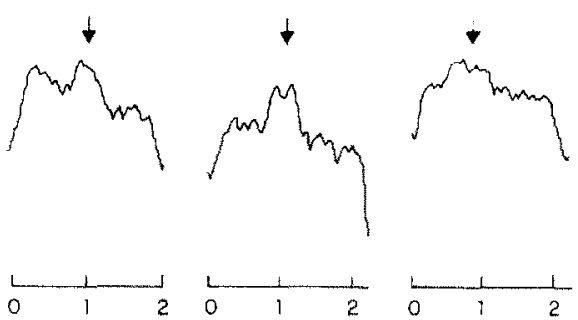

DISTANCE (mm) VM-OL

Fig. 4. (Upper panel): Typical patterns of 2-DG uptake evoked with a bipolar prosthesis in the basal turn, approximately $2 \mathrm{~mm}$ above the round window, in the posterior $\mathrm{CN}$ and IC. The separation between the two electrodes was $0.5 \mathrm{~mm}$. They were oriented longitudinally just beneath the osseous spiral lamina. (Lower panel): Optical density scans along the tonotopic axis of the IC after stimulation with a bipolar cochlear prosthesis at stimulus levels of 25 to $400 \mu \mathrm{A}$. The bipolar prosthesis produced a localized peak at all suprathreshold currents. The peak at $200 \mu \mathrm{A}$ shows two distinct maxima. 


\section{BIPOLAR PROSTHESIS}

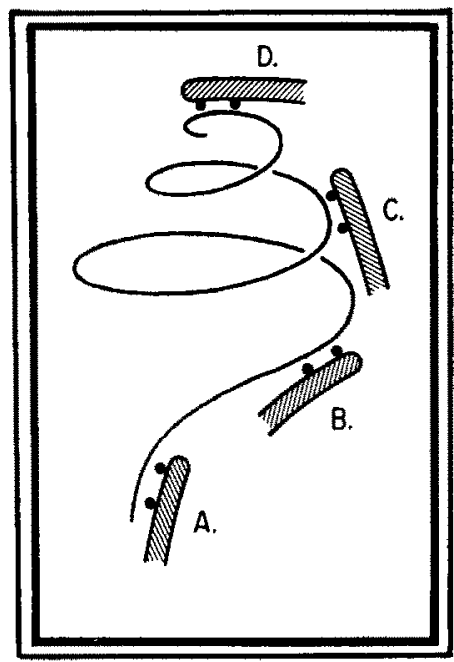
A. $\frac{\mu A}{50}$
B. -50
C. -100
D. -100

B.
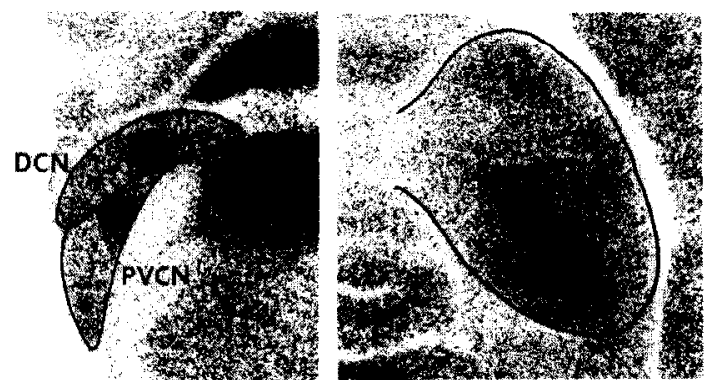

C.
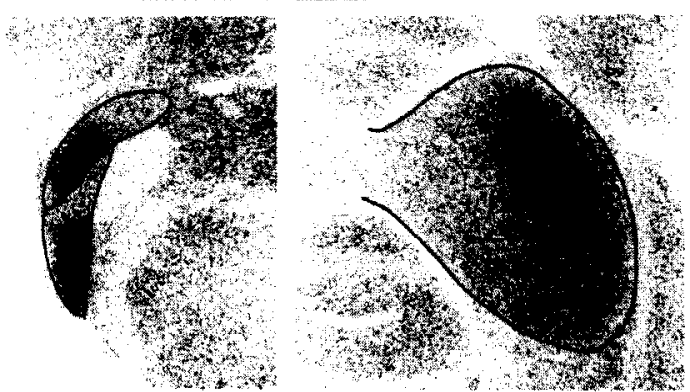

0.
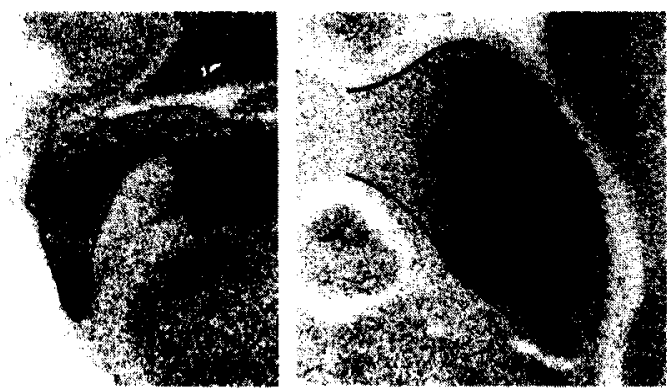

Middle

Apex
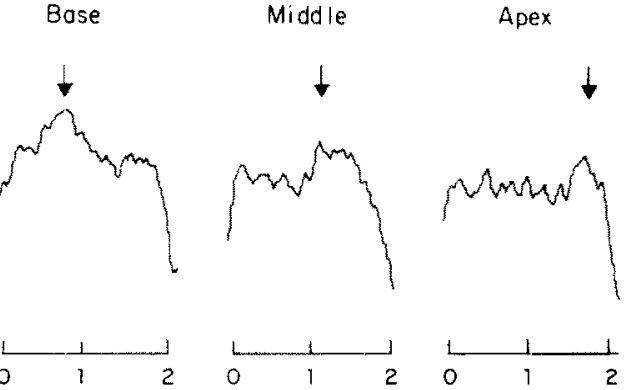

DISTANCE $(\mathrm{mm}) \cup M-D L$

Fig. 5, (Upper panel): Typical patterns of 2-DG uptake evoked with a bipolar cochlear prosthesis of four cochlear locations, at a current amplitude just above threshold, in the posterior $\mathrm{CN}$ and IC. (Lower panel): Optical density scans along the tonotopic axis of the IC after stimulation with a bipolar prosthesis at four different cochlear locations. The locus of the peak of evoked 2-DG uptake varied with prosthesis placement.

activated throughout the ventral halves of the DCN and PVCN, and the dorsal half of the IC.

The lower panel of Fig. 5 illustrates optical density profiles obtained in the IC following stimulation with a bipolar prosthesis at various cochlear locations. The region of evoked activity varied systematically with prosthesis location.

As with the monopolar prosthesis, no activity 


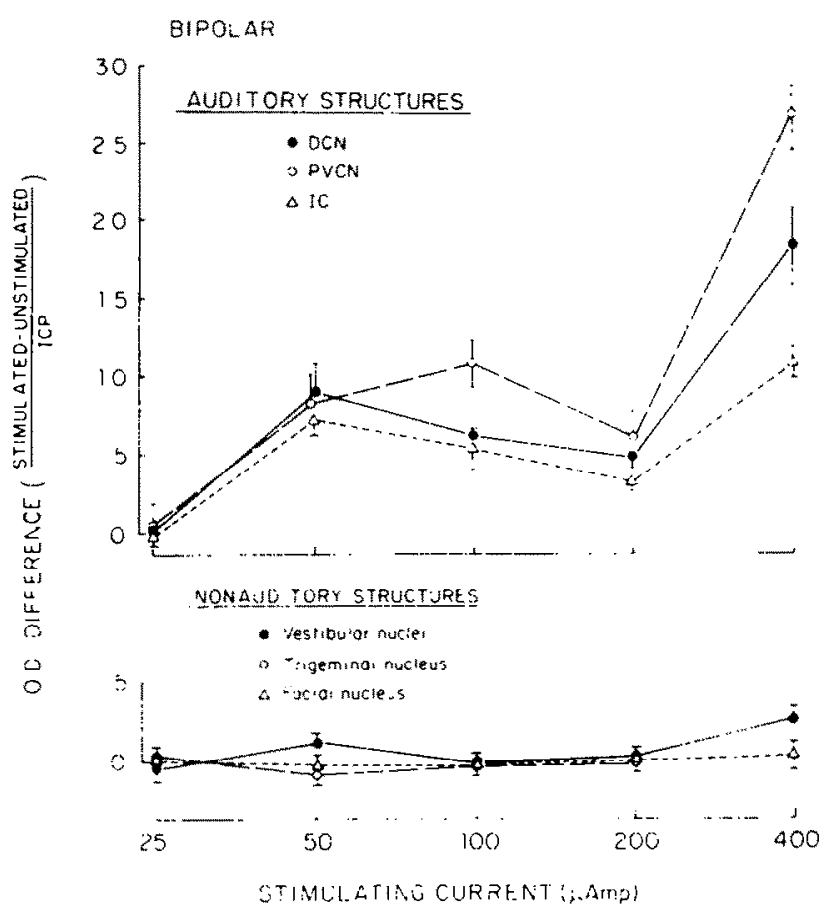

Fig. 6. Evoked 2-DG uptake produced by stimulation with a bipolar cochlear prosthesis at five current levels. The data are expressed as the optical density difference measured between each nucleus on the stimulated and unstimulated sides. (Upper panel): In three auditory nuclei, evoked uptake reached a plateau at $50 \mu \mathrm{A}$, and then increased sharply again at $400 \mu \mathrm{A}$. (Lower panel): A bipolar prosthesis increased uptake in the $\mathrm{VN}$ only at $400 \mu \mathrm{A}$.

was evoked in the medial geniculate nucleus or auditory cortex by electrical stimulation of the cochlea with a bipolar prosthesis.

The upper panel of Fig. 6 shows normalized optical density measurements of 2-DG uptake in auditory nuclei after stimulation with a bipolar prosthesis at five current levels. Stimulation at 25 $\mu \mathrm{A}$ had no effect. However, relative 2-DG uptake increased significantly $(P<0.05)$ on the stimulated side at $50 \mu \mathrm{A}$. Results obtained at 100-200 $\mu \mathrm{A}$ were similar. However, at $400 \mu \mathrm{A}$, a large increase in optical density was observed in all three auditory nuclei. Only at $400 \mu \mathrm{A}$ did relative 2-DG uptake approach the levels seen with a monopolar prosthesis at much lower currents.

The lower panel of Fig. 6 shows the responses of three non-auditory nuclei: the VN, TN and FN, to stimulation with a bipolar prosthesis. Such stimulation had little effect upon 2-DG uptake in the VN, TN or FN. Only at $400 \mu \mathrm{A}$ was a small but significant $(P<0.05)$ increase in relative $2-\mathrm{DG}$ uptake observed in the $\mathrm{VN}$.

Table I illustrates the percentage of the areas of the DCN and IC which were activated by basal turn stimulation with a bipolar prosthesis, measured from sections at the center of the A-P extent of each nucleus. Activity spread much more gradually with a bipolar than with a monopolar prosthesis, and never reached the levels of spread observed with a monopolar prosthesis at currents over $50 \mu \mathrm{A}$.

\section{Discussion}

The uptake of 2-DG, a reflection of brain metabolism, has been shown in many studies to be closely related to excitatory neuronal activity (e.g. Sokoloff, 1977; Teurich et al., 1984). The relationship between inhibitory activity and 2-DG uptake is less clear. Studies have shown both increases (Nudo and Masterton, 1986) and decreases (Ackerman, 1984) in 2-DG uptake produced by the activation of purely inhibitory inputs. Similarly, while some studies suggest that increased 2-DG uptake is primarily a synaptic (Nudo and Masterton, 1986) or primarily somatic (Durham et al., 1981) phenomenon, others indicate that evoked increases can occur in pure fiber tracts (Ryan et al., 1982). (For more detailed discussions of these issues, see Ryan and Sharp, 1982; Ryan et al., 1988). For the purposes of the present study, we interpret 2-DG uptake as reflecting evoked neural activity in a broad sense, without distinguishing between excitatory and inhibitory inputs, or sites of action at the cellular level. The possibility that insertion and activation of a cochlear prosthesis in an acute experiment would produce an alteration in brain activity unrelated to neural activity must also be considered, but this seems unlikely.

Pure tone, acoustic stimulation produced patterns of 2-DG uptake in the tissues of the IC which are consistent with many electro-physiological (e.g., Rose et al., 1963) and previous 2-DG (e.g., Huang and Fex, 1986; Ryan et al., 1982; Serviere et al., 1984; Webster et al., 1978) studies of this nucleus. These patterns reflect the spatial separation of acoustic frequencies in the cochlea, 
and the cochleotopic organization of the central auditory pathway.

Many of the effects of electrical stimulation also reflect the orderly projection of neurons from the cochlea to brainstem. Thus changes in the site of stimulation with a bipolar prosthesis produced systematic changes in the location of activated neural tissue in central auditory nuclei. Also, electrically evoked 2-DG uptake was observed in the same areas of auditory nuclei which are activated by acoustic stimulation. For example, the dorsal cortex of the IC was not activated by either electrical or sound stimulation. This suggests that neural activity evoked by electrical stimulation of the cochlea ascends the central auditory pathway by essentially the same anatomical pathways as that evoked by sound.

Clearly, the response to stimulation with a monopolar prosthesis was similar to that observed with pure tones only at the lowest intensities. However, higher amplitudes of stimulation produced responses similar to those seen with wide band noise (Sharp et al., 1981; Wang et al., 1987). Stimulation with a bipolar prosthesis produced activity which was more comparable to tonal stimulation at all amplitude levels tested.

Van den Honert and Stypulkowski $(1984,1987)$ observed little restriction of activation of VIIIth nerve fibers with a monopolar prosthesis. With bipolar, longitudinally-arranged stimulating electrodes, as used in the present study, some restriction was observed. The greatest degree of restriction was observed with bipolar, radially-oriented electrodes. However, the current densities required to activate auditory nerve fibers with radial electrodes approached the threshold for electrically induced damage.

In our study, restricted activation of IC tissue with a longitudinally arranged bipolar prosthesis observed was well maintained at high but safe current levels.

Our results are consistent with studies by Spelman et al. (1982) and Van den Honert and Stypulkowski (1984), which suggest that electrode placements which minimize shunting of current by the cochlear fluids are necessary for the restriction of electrically-evoked activity. This necessity must be balanced against the need to avoid high current densities, which can produce tissue damage.
In a previous investigation (Ryan et al., 1988) we showed that increases in intensity of a pure tonal stimulus lead to increasing volume and degree of activation of neural tissue. This spread of activation occurred toward regions which respond best to higher stimulus frequencies. Even at very high acoustic intensities, there was little spread of activity toward lower frequency regions. This is consistent with our understanding of cochlear mechanics, in which increasing tone intensity would be expected to activate more basal regions of the cochlea.

With a bipolar prosthesis, spread of activity was much more symmetrical, consistent with symmetrical spread of current around the electrode pair. In some instances, preferential spread of activity toward lower frequency regions was observed (Figs. 2A and 5C). This probably reflects spread of current into the modiolus, rather than preferential spread along or across the cochlear scalae toward the apex.

The multiple bands of 2-DG uptake observed at higher intensities of electrical stimulation in the IC (Fig. 4) may reflect local gradations in current along the basilar membrane. However, we have also observed multiple bands in the IC with high intensity pure tone (Ryan et al., 1988) or wide band noise (Wang et al., 1987) stimulation, which may be related to lateral suppression of neural activity (Ryan et al., 1988). This could also contribute to banding seen with electrical stimulation.

Both mono- and bipolar electrical stimulation at $25 \mu \mathrm{A}$ never yielded evoked 2-DG uptake, while $50 \mu \mathrm{A}$ almost always produced responses. Thus the threshold for an evoked 2-DG response to electrical stimulation was somewhere between 25 and $50 \mu \mathrm{A}$. This is higher than behavioral thresholds of about 3-10 $\mu \mathrm{A}$ reported for $1000 \mathrm{~Hz}$ sinusoids by Pfingst et al. (1984). However, thresholds for evoked 2-DG uptake to acoustic stimulation (Ryan et al., 1988) are about $25 \mathrm{~dB}$ higher than behavioral thresholds (Ryan, 1976). Finally, our 2-DG thresholds are similar to electrophysiological thresholds reported in several studies at this frequency of stimulation, as reviewed by Clopton et al. (1983).

Our optical density data suggest that the dynamic range of auditory CNS activation with a monopolar cochlear prothesis is small. Changes of 
less than $10 \mathrm{~dB}$ above threshold resulted in saturation of the level of evoked neural activity. In contrast, the response to a bipolar prosthesis (Fig. 6) displayed a plateau of response at 50,100 and $200 \mu \mathrm{A}$, but renewed growth of metabolic response at $400 \mu \mathrm{A}$. (It should be noted that we measured optical densities only of the responding tissue in each nucleus, so that the measure would not be contaminated by the extent of evoked neural activity within a nucleus. Thus the data reflect a true increase in metabolism, and presumably of neural activity, not simply spread of activity to a wider area.) This two-phase growth of 2-DG uptake suggests a much wider, although distinctly nonlinear, dynamic range.

Electrophysiological studies of VIIIth individual nerve fibers show a rapid saturation of neural responses to either monopolar (Van den Honert and Stypulkowski, 1984) or bipolar (Javel et al., 1987) electrical stimulation, with no evidence of a second growth phase. The former study also reports a narrow range of electrical thresholds for different units, along the entire cochlea for monopolar electrodes and close to the electrode pair for bipolar studies. These single unit studies suggest that saturation of population responses in the VIIIth nerve should occur over a range of about 10-15 dB. This figure agrees with our optical density observations for monopolar electrodes and for bipolar electrodes below $400 \mu \mathrm{A}$. Thus many of our observations are predictable from the responses of individual villth nerve fibers. However, the behavior of VIIIth nerve fibers cannot explain either the difference in the level of evoked metabolism observed between monopolar and bipolar stimulation, or the two-phase growth of neural activity with bipolar stimulation.

These phenomena may represent a spatial summation effect in the central auditory pathway. The 2-DG data suggest that electrical stimulation of broad regions of the cochlea activates more endings and neurons at a given frequency location in central auditory nuclei than are activated by stimulation of a corresponding narrow frequency region of the cochlea. That is, because a greater length of the cochlea is activated, more converging inputs to a given central neuron are activated, resulting in higher levels of central neural activity.
This latter possibility is supported by the observation that spread of electrical current, as evidenced by activation of $\mathrm{VN}$, was positively correlated with high levels of evoked 2-DG uptake in auditory nuclei. While VN was activated by a monopolar prosthesis from 50 to $400 \mu \mathrm{A}$, it was only activated at $400 \mu \mathrm{A}$ with a bipolar prosthesis. Similarly, only at $400 \mu \mathrm{A}$ did a bipolar prosthesis activate auditory nuclei to the extent seen with the monopolar prosthesis from $50-400 \mu \mathrm{A}$.

With a monopolar cochlear prosthesis, and especially at high current levels, activation of non-auditory structures was observed. VN activation at $50 \mu \mathrm{A}$ (Fig. 3) is difficult to reconcile with the fact that the activation of auditory nuclei at this current was restricted to a narrow frequency band (Fig. 2). Perhaps vestibular nerve fibers have lower thresholds than auditory structures. Alternatively, current may have spread to the saccule. At higher current levels, in which the entire auditory nerve was stimulated, activation of the $V N$ and TN was extensive. Lesser activation of the $F N$ was also seen. These results are consistent with the expected wider spread of current from a monopolar current source. The vestibular and facial nerve roots run in the internal auditory meatus, and thus might have been activated by current at that level. However, while portions of the trigeminal nerve travel through the middle ear, the great majority of this nerve does not travel near the cochlea. It is therefore significant that the $\mathrm{TN}$ were activated to a greater extent than the FN. This could be accounted for by threshold differences between the fibers of the two nerves. However, it seems more likely that the TN and FN were activated by current which reached them at the brainstem level, rather than at their peripheral nerves, since the TN are closer to the current source than the FN.

The idea of current spread through the brainstem is supported by the recent observations of Evans et al. (1990), who measured 2-DG uptake in guinea pigs during electrical stimulation of the $\mathrm{CN}$ with a monopolar electrode. They found widespread activation of auditory structures and the vestibular nuclei, similar to our results with monopolar cochlear stimulation. However, in contrast to cochlear stimulation, $\mathrm{CN}$ stimulation 
activated both sides of the brain, not just the stimulated side. This suggests extensive spread of activity from the electrode site.

\section{Acknowledgements}

Supported by the Research Service of the Veterans Administration, by NIH grants DC00139 and NS21440, and by the Duaei Hearing Research Fund. Dr. Elizabeth Keithley provided invaluable critical comments on the manuscript.

\section{References}

Ackerman, S.K. (1984) Increased glucose metabolism during long-duration recurrent inhibition of hippocampal pyramidal cells. J. Neurosci, 4, 251-264.

Clopton, B.M., Spelman, F.A., Glass, I., Pfingst, B.E., Miller, J.M., Lawrence, P.D. and Dean, D.P. (1983) Neural encoding of electrical signals. Ann. N. Y. Acad. Sci. 405, 146-158.

Durham, D., Woolsey, T. and Kurger, L. (1981) Cellular localization of $2-[3 \mathrm{H}] \mathrm{deoxy}-\mathrm{D}$-glucose from paraffin-embedded brains. J. Neurosci. 1, 519-526.

Evans, D.A., Niparko, J.K., Altschule, R.A., Frey, K.A. and Miller, J.M. (1990) Demonstration of prosthetic activation of central auditory pathways using $\left[{ }^{14} \mathrm{C}\right]-2$-deoxyglucose. Laryngoscope 100, 128-137.

Glass, I. (1983) Tuning characteristics of cochlear nucleus units in response to electrical stimulation. Hear. Res. 12, 223-237.

Huang, C. and Fex, J. (1986) Tonotopic organization in the inferior colliculus of the rat demonstrated with the 2-deoxyglucose method. Exp. Brain Res. 61, 506-512.

Hungerbuhler, J.P., Saunders, J.C., Greenberg, J. and Reivich, M. (1981) Functional neuroanatomy of the auditory cortex studied with $\left(2-{ }^{14} \mathrm{C}\right)$ deoxyglucose. Exper. Neurol. 71. 104-121.

Javel, E., Tong, Y.C., Shepherd, R.K. and Clark, G.M. (1987) Responses of cat auditory nerve fibers to biphasic electrical current pulses. Ann. Otol. Rhinol. Laryngol. 96, Suppl. 128, $26-30$.

Merzenich, M.M. and White, M. (1977) Cochlear implant. The interface problem. Biomed. Eng. Instrum. Funct. Electr. Stimul. 3, 321-340.

Nudo, R.J. and Masterton, R.B. (1986) Stimulation-induced $\left({ }^{14} \mathrm{C}\right) 2$-deoxyglucose labeling of synaptic activity in the central auditory system. J. Comp. Neurol. 245, 553-565.

Pfingst, B.E., Glass, I., Spelman, F.A. and Sutton, D. (1984) Psychophsyical studies of cochlear implants in monkeys: Clinical implications. In: R.A. Schindler and M.M. Merzenich (Eds.), Tenth Anniversary Conference on Cochlear Implants: An International Symposium. Raven Press, New York.

O'Leary, S.J., Black, R.C. and Clark, G.M. (1985) Current distributions in the cat cochlea: A modeling and electrophysiological study. Hear. Res. 18, 273-281.
Ostapoff, E.-M. and Morest, D.K. (1989) A degenerative disorder of the central auditory system of the gerbil. Hear Res. 37, 141-162.

Rose, J.E., Greenwood, D.D., Goldberg, J.M. and Hind, J.E. (1963) Some discharge characteristics of single neurons in the inferior colliculus of the cat. 1. Tonotopical organization, relation of spike-counts to tone intensity, and firing patterns of single elements. $J$. Neurophysiol. $26,294-320$.

Ryan, A. (1976) Hearing sensitivity of the mongolian gerbil, Meriones unguiculatus. JASA 59, 1222-1226.

Ryan, A.F., Woolf, N.K. and Sharp, F.R. (1982) Tonotopic organization in the central auditory pathway of the mongolian gerbil: a 2-deoxyglucose study. J. Comp. Neurol. 207, 369-380.

Ryan, A.F. and Sharp, F.R. (1982) Localization of [3H]2-deoxyglucose at the cellular level using freeze-dried tissue and dry-looped emulsion. Brain Res. 252, 177-180.

Ryan, A.F. and Woolf, N.K. (1988) Development of tonotopic representation in the central auditory system of the mongolian gerbil: a 2-deoxyglucose study. Devel. Brain Res. 41, 61-70.

Ryan, A.F., Braverman, S., Woolf, N.K. and Axelsson, G.A. (1988) Auditory neural activity evoked by pure-tone stimum lation as a function of intensity. Brain Res. 483, 293-203.

Scheich, H., Heil, P. and Langner, G. (1986) Tonotopic organization of gerbil auditory cortices mapped with 2-deoxyglucose. Neurosci. Abstr. 12, 1274.

Schmeidt, R.A. (1977) Single and two-tone effects in normal and abnormal cochleas: a study of cochlear microphonics and auditory-nerve units. Ph.D. Thesis, Syracuse University.

Serviere, J., Webster, W.R. and Calford, M.B. (1984)Isofrequency labeling revealed by a combined $\left[1^{14} \mathrm{C}\right]-2$-deoxyglucose, electrophysiological, and horseradish peroxidase study of the inferior colliculus of the cat. J. Comp. Neurol. 228, 463-477.

Sharp, F.R., Kilduff, T.S., Bzorgchami, S. Heller, H.C. and Ryan, A.F. (1983) The relationship of local cerebral glucose utilization to optical density ratios. Brain Res. 263, 97-103.

Sharp, F.R., Ryan, A.F., Goodwin. P. and Woolf, N.K. (1981) Increasing intensities of wide-band noise increase ${ }^{14}-\mathrm{C}-2$ deoxyglucose uptake in gerbil central auditory structures. Brain Res. 230, 87-96.

Sokoloff, L. (1977) Relation between physiological function and energy metabolism in the central nervous system. I. Neurochem. 29. 13-26.

Spelman, F.A., Clopton, B.M. and Pfingst, B.E. (1982) Tissue impedance and current flow in the implanted ear. Implications for the cochlear prosthesis. Ann. Otol. Rhinol. Laryngol. 91. Suppl. 98, 3-8.

Theurich, M., Muller, C.M. and Scheich, H. (1984) 2-deoxyglucose accumulation parallels extracellularly recorded spike activity in the avian auditory neostriatum. Brain Res. $322,157-161$.

Van den Honert, C. and Stypulkowski, P.H. (1984) Physiological response properties of the electrically stimulated auditory nerve. II. Single fiber recordings. Hear. Res. 14, 225 243. 
Van den Honert, C. and Stypulkowski, P.H. (1987) Single fiber mapping of spatial excitation patterns in the electrically stimulated auditory nerve. Hear. Res. 29, 195-206.

Wang, Z.X., Ryan, A.F. and Woolf, N.K. (1987) Pentobarbital and ketamine on alter 2-deoxyglucose uptake pattems in the central auditory system of the gerbil. Hear. Res. 27. 145-155.
Webster, W.R., Serviere, J., Batini, C. and Lapiante, S. (1978) Autoradiographic demonstration with $2-\left({ }^{14} \mathrm{C}\right)$-deoxyglucose of frequency selectivity in the auditory system of cats under conditions of functional activity, Neurosci. Letters 10 (1978) $43-48$. 